\title{
Acute heart failure due to COVID-19 related myocardial injury and de novo hypertensive cardiomyopathy: a challenging diagnosis
}

\author{
Matteo Pernigo', Marco Triggiani' ${ }^{1}$, Emanuele Gavazzi ${ }^{2}$, Ilaria Papa ${ }^{1}$, Alberto Vaccari ${ }^{1}$, Simona Fisogni ${ }^{3}$, \\ Ester Costantino ${ }^{4}$, Gian Franco Pasini ${ }^{1}$ \\ ${ }^{1}$ Cardiology Unit, 'La Memoria' Hospital, Gavardo (BS); ${ }^{2}$ Institute of Radiology, Department of Medical and surgical \\ Specialties, University of Brescia; ${ }^{3}$ Institute of Anatomical Pathology, Department of Medical and Surgical Specialties, \\ University of Brescia; ${ }^{4}$ Nephrology Unit, 'La Memoria' Hospital, Gavardo (BS), Italy
}

\begin{abstract}
We report a COVID-19 case with acute heart and kidney failure in a healthy young male. Echocardiography showed severe systolic and diastolic left ventricle dysfunction, with diffuse myocardial thickening. Cardiac MRI showed aspects of focal myocarditis, and
\end{abstract}

Correspondence: Matteo Pernigo, MD. Cardiology Unit, 'La Memoria' hospital, via Andrea Gosa 74, Gavardo (BS), Italy.

E-mail: matteo.pernigo@gmail.com

Key words: COVID-19; myocarditis; cardiac MRI; hypertensive cardiomyopathy; echocardiography.

Contributions: MP, MT, drafted the manuscript; EG, SF, EC, GFP, took part to clinical management of the case and data collection; IP, $\mathrm{AV}, \mathrm{SF}, \mathrm{EG}$, revised the manuscript. All the authors have read and approved the final version of the manuscript and agreed to be accountable for all aspects of the work

Conflict of interest: The authors declare that they have no competing interests, and all authors confirm accuracy.

Availability of data and materials: All data underlying the findings are fully available.

Ethics approval and consent to participate: No ethical committee approval was required for this case report by the Department, because this article does not contain any studies with human participants or animals. Informed consent was obtained from the patient included in this study.

Consent for publication: The patient gave his written consent to use his personal data for the publication of this case report and any accompanying images.

Received for publication: 23 January 2021.

Accepted for publication: 28 July 2021.

${ }^{\circ}$ Copyright: the Author(s), 2021

Licensee PAGEPress, Italy

Monaldi Archives for Chest Disease 2022; $92: 1778$

doi: $10.4081 /$ monaldi.2021.1778

This article is distributed under the terms of the Creative Commons Attribution Noncommercial License (by-nc 4.0) which permits any noncommercial use, distribution, and reproduction in any medium, provided the original author(s) and source are credited. hypertensive cardiomyopathy. Renal biopsy demonstrated limited acute tubular injury, and hypertensive kidney disease. Coronary angiography excluded critical stenoses. Unlike what we initially suspected, myocardial inflammation had a limited extent in our patient; severe hypertension causing cardiomyopathy and multiorgan damage, not diagnosed before, was primarily responsible for severe illness. Correct diagnosis and guidelines-directed treatment allowed a favorable course.

\section{Introduction}

Coronavirus disease 2019 (COVID-19) is at the moment an international public health emergency, caused by a novel enveloped RNA beta-coronavirus, named 'severe acute respiratory syndrome coronavirus-2' (SARS-CoV-2). The infection is mainly characterized by respiratory tract symptoms, presenting as mild flulike illness to potentially lethal acute respiratory distress syndrome or fulminant pneumonia, but relevant cardiovascular complications and multi-organ failure can occur in severe cases [1]. Patients with known cardiovascular disease and risk factors appear more vulnerable to the infection, and hypertension is highly prevalent in patients who develop severe illness. Pathological mechanism underlying this association in still not completely elucidated: binding of the virus to angiotensin-converting enzyme 2 receptor (ACE-2), highly expressed on endothelial surfaces, with consequent renin-angiotensin-aldosterone system deregulation, and cytokine storm seem to have a relevant role [2].

We report a case of acute $\mathrm{HF}$ and documented cardiac inflammation during COVID-19. Multi-modality cardiac imaging and a challenging interdisciplinary medical approach led to a denovo diagnosis of hypertensive multi-organ damage, exacerbated by concomitant viral infection.

\section{Case Report}

A 45-year-old man presented to our Emergency Department in April 2020 for atypical chest pain, fever and worsening effort dyspnoea from 7 days before. His past medical history was unremarkable and he denied alcohol and/or drugs abuse. On his arrival high blood pressure $(\mathrm{BP}=180 / 110 \mathrm{mmHg}$ ), bi-basal pulmonary rales and mild leg swelling were detected; oxygen saturation was $93 \%$ while breathing ambient air. Electrocardiography showed sinus tachycardia with left axis 
deviation, slight diffuse ST depression more prominent in inferiorlateral leads (Figure 1A). Blood tests revealed a significant increase in Troponin T ( $82 \mathrm{ng} / \mathrm{L}$; being $14 \mathrm{ng} / \mathrm{L}$ the upper reference limit of normal - URL), serum creatinine $(3.1 \mathrm{mg} / \mathrm{dl}$; eGFR $20 \mathrm{ml} / \mathrm{min}$ $\left.\mathrm{ml} / \mathrm{min} / 1.73 \mathrm{~m}^{2}\right)$ and C-reactive protein $(30 \mathrm{mg} / \mathrm{L}$; being $5 \mathrm{mg} / \mathrm{L}$ the URL). At blood cell count, neutrophils were slightly augmented and lymphocytes slightly diminished. Nasopharyngeal swab was positive for SARS-CoV-2 infection. Chest X-rays and High resolution computed tomography scan of the lungs showed ground glass-like hyper density opacities limited to the peripheral portions of inferior lobes bilaterally, and a small area of consolidation in the left superior lobe (Figure 1 B,C). Transthoracic echocardiography was also performed and showed mild increase in LV dimension (end diastolic diameter: $57 \mathrm{~mm}$; end diastolic volume: $141 \mathrm{ml}, 83 \mathrm{ml} / \mathrm{m} 2$ BSA) and moderate increase in walls thickness (interventricular septum $=13.5 \mathrm{~mm}$; posterior wall $=11 \mathrm{~mm}$ ), with a sparkling appearance. LV ejection fraction (LVEF) was severely reduced because of a diffuse parietal hypokinesis $(\mathrm{LVEF}=30 \%$; quantified by Simpson's method). Left atrium was moderately enlarged (volume $=73 \mathrm{ml}, 43 \mathrm{ml} / \mathrm{m}^{2} \mathrm{BSA}$ ), and the mitral diastolic filling pattern was restrictive. There was a mild mitral and tricuspid regurgitation and a mild pericardial effusion was noted behind the inferior-lateral LV wall. A few hours after admission to our cardiac intensive care unit, patient's condition degenerated abruptly; he presented BP elevation to $210 / 130 \mathrm{mmHg}$ and respiratory failure, requiring nitroglycerine infusion, high doses of intravenous furosemide and non-invasive ventilatory support with continuous positive airway pressure (CPAP) of $10 \mathrm{~cm} \mathrm{H20.} \mathrm{From} \mathrm{the} \mathrm{next} \mathrm{days,}$ clinical conditions began to improve, allowing removal of CPAP on day 3. Therapy with bisoprolol was initiated and gradually titrated up to $3.75 \mathrm{mg} / \mathrm{die}$; amlodipine $10 \mathrm{mg} / \mathrm{die}$ and alfuzosin $2 \mathrm{mg}$ bid were also introduced for BP control. For viral infection, hydroxychloroquine (200 mg bid) and darunavir/ritonavir (800/100 $\mathrm{mg}$ od) were administered for 10 and 5 days respectively according to local protocol. Feverish episodes (temperature $>38^{\circ} \mathrm{C}$ ) persisted for the first 4 days. Clinical laboratory tests trend during hospitalization is summarized in Table 1. Notably, serum creatinine remained constantly elevated; there was modest proteinuria (228 $\mathrm{mg} / \mathrm{L}$ ), without Bence-Jones proteinuria; serum and urine protein immunofixation excluded monoclonal free light chains.

When patient's clinical conditions were stable, echocardiogram was repeated and confirmed diffuse parietal hypokinesis with improvement in LV systolic and diastolic function (LVEF $=38 \%$ and 'pseudonormal' diastolic filling pattern) (Figure 2). Diffuse myocardial thickening was still evident. 2D Speckle-tracking showed diffuse reduction of global longitudinal strain, more severe in anterior segments, and partial apical sparing. Cardiac magnetic resonance imaging (MRI) was performed, showing increased symmetric and circumferential wall thickness, with diffuse LV hypokinesis (LVEF 35\%) and mild hypokinesis of right ventricle (RVEF 41\%). Short tau inversion recovery and T2-Mapping sequences demonstrated the presence of focal myocardial oedema, in medium and apical segments of anterior wall and anterior septum. Phase-sensitive inversion recovery sequences showed a diffuse and intramural late gadolinium enhancement (LGE) of LV myocardium in all segments, with circumferential distribution. Pericardial effusion was not present (Figure 3). This pattern resulted appeared consistent with hypertensive cardiomyopathy, and myocarditis of
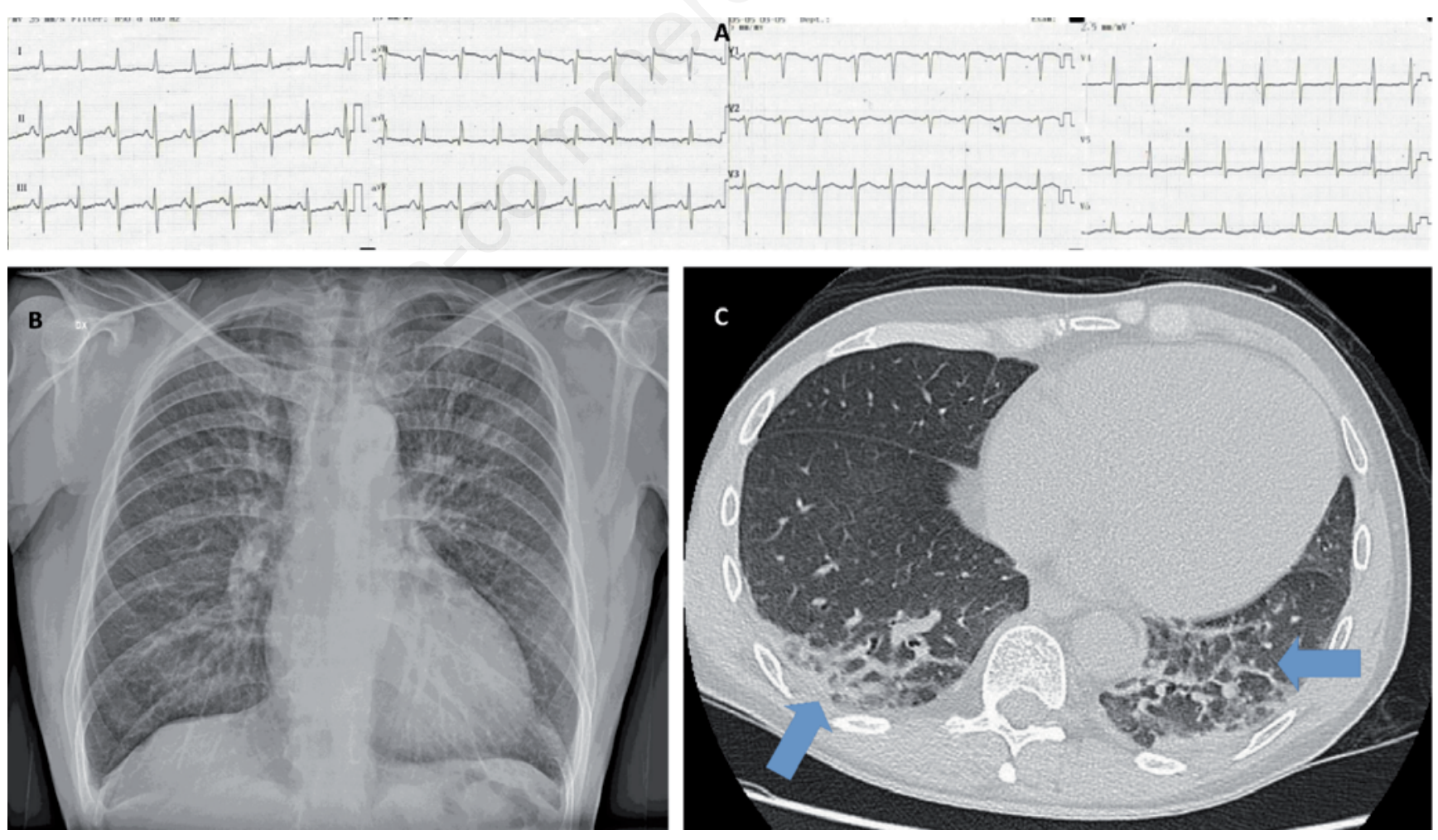

Figure 1. A) Electrocardiogram on admission. Sinus tachycardia with left axis deviation, slight diffuse ST depression more prominent in inferior-lateral lead. B) Chest X-ray. Interstitial oedema limited to left superior and lateral regions, and no pleural effusion. C) Image from high resolution computed tomography scan of the lungs. Ground glass-like hyper density opacities in the peripheral portions of inferior lobes bilaterally (arrows). 
limited extent. The day after, the patient underwent cranial computed tomography, showing multiple ischemic lacunar foci of the basal ganglia bilaterally, a stabilized ischemic lesion of the right corona radiata and white matter hypodensity (Supplementary figure 1). Doppler ultrasound excluded clinically relevant carotid stenosis, and significant renal arteries pathology. Renal biopsy demonstrated diffuse acute tubular injury and modest chronic interstitial inflammation, with minimal tubulitis. Severe arteriolar hyalinosis (also in preglomerular arterioles), glomerular capsular thickening and capillary basement membrane wrinkling and duplication were also present, as for chronic hypertensive kidney disease (Figure 4). Amyloid deposits were excluded. Fifteen days after admission, the patient was discharged. One month later, after clinical and biochemical resolution of COVID-19 as demonstrated by two consecutive negative nasopharyngeal swabs, he was readmitted in our Unit for coronary angiography control. This showed diffuse atherosclerotic disease of epicardial vessels without critical stenoses (Supplementary Figure 2). A new echocardiogram demonstrated further reduction of LV volumes with improvement in $\mathrm{EF}$ (to $46 \%$ ). Assessment of left ventricular filling demonstrated an abnormal relaxation pattern (grade I diastolic dysfunction). Renal function was persistently impaired (serum creatinine: $2.9 \mathrm{mg} / \mathrm{dl}$ ). Cardiac MRI performed 2 months later, showed a reduction in biventricular volumes with improvement in LVEF up to $50 \%$; LV parietal hypertrophy was unchanged (septal thickness: $13 \mathrm{~mm}$ ) with mild, diffuse intramural LGE; T1 and T2 sequences showed oedema resolution.

\section{Discussion}

The present report describes a case of COVID 19 with acute respiratory failure, acute myocardial and kidney injury, in a patient firstly diagnosed with severe hypertension causing dilated cardiomyopathy and multiple target-organ damage. Definite diagnosis was possible through myocardial and renal tissue characterization. Notably, they were both obtained in a living patient with severe COVID 19, the first through cardiac magnetic resonance, the latter by means of kidney biopsy. Pre-existing CV disease were found to be independent predictor of in-hospital mortality in COVID-19 patients [1-3]. Moreover, severe illness may be complicated by acute myocardial injury, clinically expressed by electrocardiographic abnormalities, myocardial dysfunction and troponin release, being associated with a worse prognosis $[4,5]$. Cardiac involvement may occur due to ischemic (acute myocardial ischemia/infarction) or non-ischemic processes, including stressinduced cardiomyopathy and myocarditis.

Several cases of acute heart failure or cardiogenic shock caused with echo-documented and MRI-proven myocardial inflammation due to COVID-19 have been published so far [6-8]; cardiac MRI showed diffuse, extensive myocardial oedema conditioning global cardiac function impairment. In our case, echocardiography during hospitalization showed diffuse myocardial thickening, with an echobright appearance, severe diastolic dysfunction with initially restrictive filling pattern, bi-atrial enlargement and mild pericardial

Table 1. Laboratory tests during hospitalization.

\begin{tabular}{|c|c|c|c|c|c|c|c|c|}
\hline Measure & Reference range & Day 1 & Day 3 & Day 5 & $\begin{array}{c}\text { Results } \\
\text { Day } 7\end{array}$ & Day 9 & Day 11 & Day 13 \\
\hline Haemoglobin, g/dL & $14.2-17.2$ & 14 & 12.6 & 12 & 11.5 & 11.9 & 12 & 12.4 \\
\hline White blood cell count, $10^{9} / \mathrm{L}$ & $4.2-9.4$ & 8.98 & 7.26 & 7.24 & 7.46 & 7.01 & 7.63 & 9.66 \\
\hline Lymphocyte relative count, \% & $19.6-46.5$ & 14.6 & 16.8 & 16.9 & 10.2 & 27.5 & 33.6 & 29.0 \\
\hline Lymphocyte absolute count, $10^{9} / \mathrm{L}$ & $1.1-3.4$ & 1.31 & 1.22 & 1.22 & 0.76 & 1.93 & 2.56 & 2.81 \\
\hline Neutrophil relative count, $\%$ & $40.6-68.4$ & 82 & 79 & 77 & 85 & 65 & 58 & 60.8 \\
\hline Neutrophil absolute count, $10^{9} / \mathrm{L}$ & $2-5.8$ & 3.2 & 5.73 & 5.58 & 6.3 & 4.57 & 4.46 & 2.91 \\
\hline Platelet count, $10^{9} / \mathrm{L}$ & $155-320$ & 273 & 224 & 197 & 186 & 267 & 353 & 500 \\
\hline D-dimer, ug/L & $<500$ & 1026 & 1865 & 875 & 889 & 863 & 821 & 795 \\
\hline Creatinine, mg/dL & $0.7-1.2$ & 3,1 & 3,03 & 3.3 & 3.2 & 2.9 & 2.7 & 2.9 \\
\hline eGFR, $\mathrm{ml} / \mathrm{min} / 1,73 \mathrm{~m}^{2}$ & & 21 & 22 & 20 & 21 & 24 & 25 & 24 \\
\hline Sodium, mmol/L & $136-145$ & 134 & 135 & 130 & 133 & 131 & 138 & 135 \\
\hline Potassium, mmol/L & $3.5-5.1$ & 2.35 & 2.5 & 2.5 & 3.1 & 3.7 & 4.3 & 4.4 \\
\hline Aspartate aminotransferase & $<40$ & 22 & & 54 & & 28 & & 43 \\
\hline Alanine aminotransferase & $<40$ & 16 & & 15 & & 19 & & 45 \\
\hline Hs-troponin T, ng/L & $<14$ & 89 & 97 & 109 & 107 & 84 & 80 & 35 \\
\hline C-reactive protein, mg/dL & $<5$ & 30 & 54 & 150 & 104 & 79 & 43 & 25 \\
\hline $\mathrm{Il}-6, \mathrm{pg} / \mathrm{ml}$ & $0-7$ & & & 47.4 & & & & 28.9 \\
\hline Serum Kappa light chains, mg/L & $3.3-19.4$ & & & & & & & 168 \\
\hline Serum Lambda light chains, mg/dL & $5.7-26.3$ & & & & & & & 98 \\
\hline Free light chain ratio & $0.26-1.65$ & & & & & & & 1.7 \\
\hline Bence Jones proteinuria & & & & & & & & Absent \\
\hline Urinary proteins, mg/L & $0-150$ & & & & & & 228 & \\
\hline Immunofixation of urine proteins & & No $\mathrm{m}$ & clonal fre & ht chains; & lar prot & & & \\
\hline Immunofixation of serum proteins & & No $m$ & clonal fre & ht chains & & & & \\
\hline
\end{tabular}




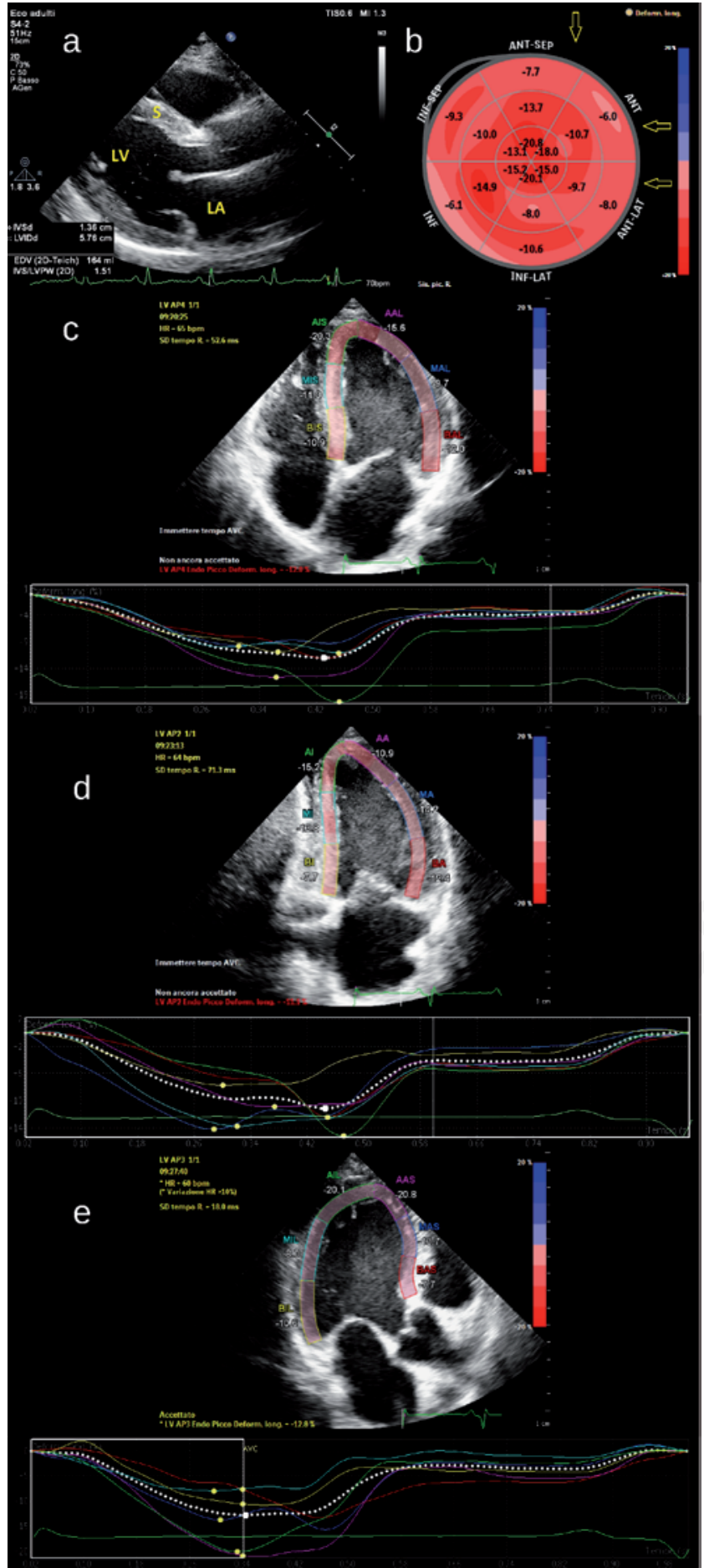

Figure 2. Echocardiogram performed on day $10^{\text {th }}$ A) Parasternal long-axis view, showing moderate concentric LV hypertrophy with echo-bright appearance of myocardium. LV, left ventricle; $S$, septum; LA, left atrium. b-e) 2D-speckle tracking longitudinal strain evaluation. b) Bull's eye with global results; global longitudinal strain: $-12 \%$ (18 segments); diffuse hypokinesia is evidenced, more pronounced in basal anterior and septal segments (arrows), and relative apical sparing. c,d,e) Longitudinal strain evaluated in apical 4-chamber, 2-chamber, and 3-chamber views, respectively, with strain curves for each segment. effusion. Moreover, global longitudinal strain with partial apical sparing was detected at 2D-speckle tracking. These aspects aroused at first the suspect of a possible infiltrative cardiomyopathy, as amyloidosis. Other diagnostic hypotheses were: extensive COVID19 related myocardial inflammation with diffuse interstitial infiltrates, or misdiagnosed hypertensive cardiomyopathy. Systemic amyloidosis was also at first suggested by the presence of kidney failure, but this hypothesis was later rejected on the basis of serum and urine immunofixation. Cardiac MRI was crucial in the diagnostic process; it confirmed diffuse myocardial thickening and systolic impairment; myocardial oedema depicted by T1 and T2 sequences had a limited extent, thus suggesting that the severe cardiac impairment was not exclusively caused by myocarditis.

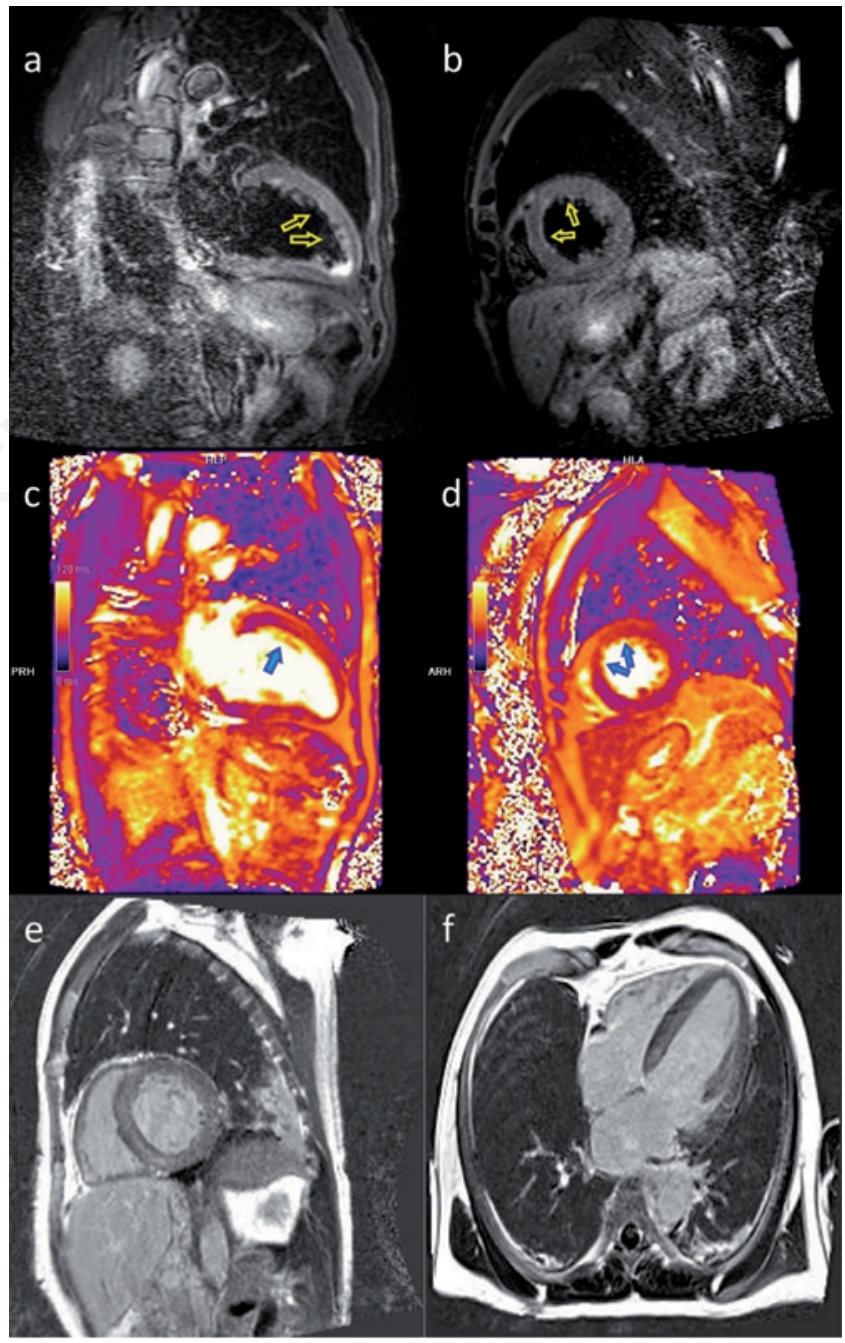

Figure 3. Cardiac magnetic resonance imaging. Short tau inversion recovery (STIR) sequences in 2-chamber (A) and short-axis view (B) showed mild myocardial signal hyperintensity in left ventricle anterior wall and anterior septum in medium and apical segments (arrows), consistent with interstitial oedema, confirmed and best depicted in corresponding T2-Mapping sequences (C and $\mathrm{D}$, arrows). Phase sensitive inversion recovery (PSIR) sequences in short-axis (E) and 4-chamber view (F) showed diffuse intramural late gadolinium enhancement. 


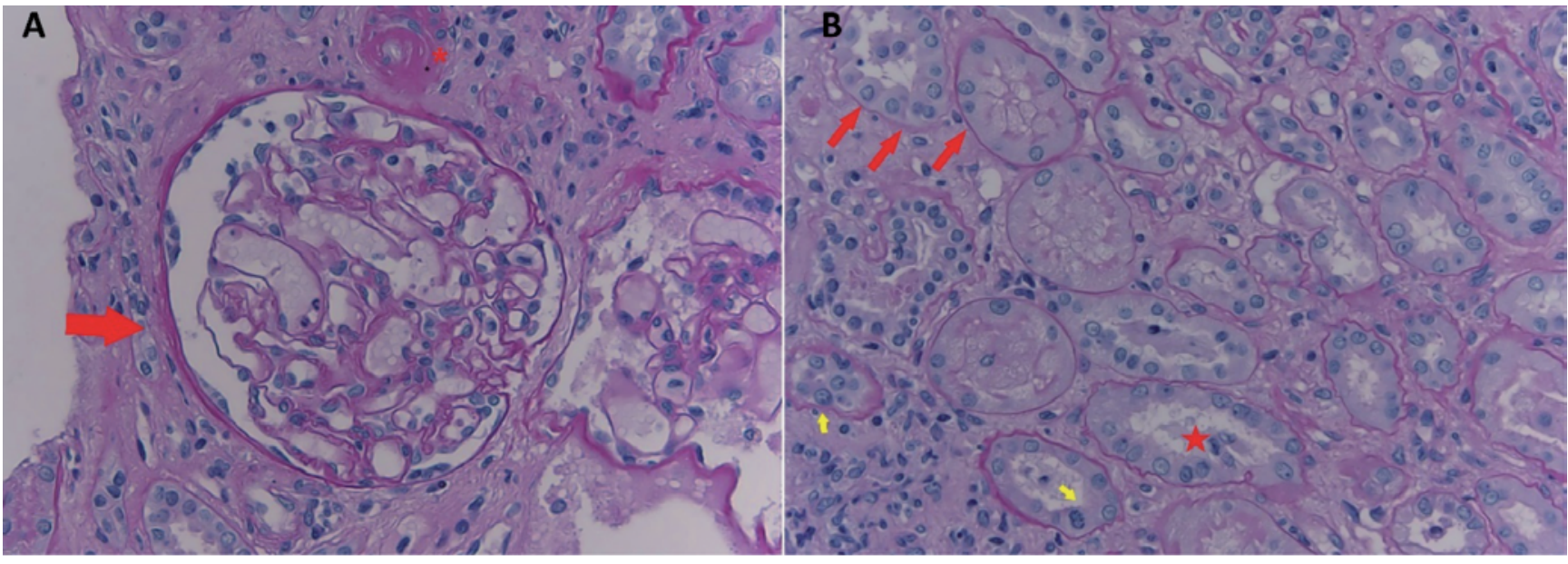

Figure 4. Renal biopsy specimens, PAS (Periodic Acid Schiff) stain. A) Capsular thickening and wrinkling of glomerular basement membrane (arrow). Circumferential arteriolar hyaline sclerosis (asterisk). B) Diffuse acute tubular injury with brush border loss, tubular dilatation, epithelial thinning or swollen, focal vacuolization (red arrows); partial desquamation of tubular epithelial cells (star); focal regeneration features (prominent nucleoli and mitosis - yellow arrows); modest interstitial chronic inflammation with minimal tubulitis.

LGE pattern appeared non suggestive for amyloidosis and supported the diagnosis of hypertensive cardiomyopathy. Interestingly, cardiac function gradually improved during and after hospitalization, in parallel with COVID-19 resolution and effective treatment of hypertension.

Kidney impairment is frequent in COVID-19 patients: more than $40 \%$ of them have proteinuria at hospital admission [9], while some critically ill patients may develop during hospitalization an acute kidney injury (AKI) [10]. The most common pathogenic factors appear related to direct renal injury (promoted by the binding of SARS-CoV-2 to ACE-2 on tubular cells and podocytes), to microthrombi formation due to hypercoagulability, and to the effects of acute systemic inflammatory response [10]. Kidney biopsy findings in our patient revealed diffuse acute tubular damage with modest interstitial inflammation, as for AKI, together with glomerular and vascular alterations consistent with chronic hypertensive nephropathy. Resolution of infective process however did not lead to a significant improvement of kidney function; thus, we can speculate that in this patient the long-time undiagnosed severe hypertension was the main contributor to his kidney impairment. Cranial CT findings, suggestive for hypertensive encephalopathy, were also in keeping with a severe uncontrolled hypertension not diagnosed before.

In conclusion, our report depicts how a condition of severe, untreated hypertension can increase cardiac vulnerability to COVID-19 infection: acute inflammatory alterations, even of limited extent, may critically destabilize a pre-existing organ damage of hypertensive aetiology, leading to a condition of severe HF. This report highlights that in these patients the same mechanistic approach to cardiac and systemic abnormalities should be used, increasing utilization of multi-modality imaging and diagnostic tools that may help us to make a correct diagnosis.

\section{References}

1. Zhou F, Yu T, Du R, et al. Clinical Course and risk factors for mortality of adult inpatients with COVID-19 in Wuhan, China: A retrospective cohort study. Lancet 2020; 395:1054-62.

2. Xiong T-Y, Redwood S, Prendergast B, Chen M. Coronaviruses and the cardiovascular system: acute and long-term implications. Eur Heart J 2020; 41:1798-800.

3. Tomasoni D, Inciardi RM, Lombardi CM, et al. Impact of heart failure on the clinical course and outcomes of patients hospitalized for COVID-19. Results of the Cardio-COVID-Italy multicentre study. Eur J Heart Fail 2020;22:2238-47.

4. Li J-W, Han T-W, Woodward M, et al. The impact of 2019 novel coronavirus on heart injury: A systemic review and metaanalysis. Prog Cardiovasc Dis 2020;63:518-24.

5. Lombardi CM, Carubelli V, Iorio A, et al. Association of troponin levels with mortality in Italian patients hospitalized with Coronavirus Disease 2019: Results of a multicenter study. JAMA Cardiol 2020;5:1274-80.

6. Inciardi RM, Lupi L, Zaccone G, et al. Cardiac involvement in a patient with Coronavirus Disease 2019 (COVID-19). JAMA Cardiol 2020;5:819-24.

7. Sala S, Peretto G, Gramegna M, et al. Acute myocarditis presenting as a reverse Tako-Tsubo syndrome in a patient with SARS-CoV-2 respiratory infection. Eur Heart J 2020 14;41:1861-2.

8. Kim IC, Kim JY, Kim HA, Han S. COVID-19-related myocarditis in a 21-year-old female patient. Eur Heart 2020;41:1859.

9. Cheng Y, Luo R, Wang K, et al. Kidney disease is associated with in-hospital death of patients with COVID-19. Kidney Int 2020; 97 829-38.

10. Ronco C, Reis T, Husain-Syed F. Management of acute kidney injury in patients with COVID-19. Lancet Respir Med 2020;8:738-42. 